\title{
Tradução e Validação da Escala FOUR para a Pediatria e o seu Uso Como Indicador Prognóstico: Um Estudo Piloto
}

\author{
Translation and Validation of the FOUR Scale for Children \\ and its Use as Outcome Predictor: A Pilot Study
}

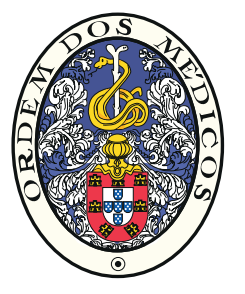

\author{
Sofia Simões FERREIRA $\triangle 1$, Daniel MEIRELES ${ }^{2}$, Alexandra PINTO ${ }^{3}$, Francisco ABECASIS ${ }^{4}$
}

Acta Med Port 2017 Sep;30(9):599-607 - https://doi.org/10.20344/amp.8052

\begin{abstract}
RESUMO
Introdução: A escala FOUR - Full Outline of UnResponsiveness, já validada na população adulta, avalia a depressão do estado de consciência. O objetivo deste estudo consiste na tradução para português e validação da escala FOUR na população pediátrica, assim como na comparação da pontuação obtida e da evolução clínica dos doentes entre a escala FOUR e a escala de coma de Glasgow, nos doentes internados numa unidade de cuidados intensivos pediátricos.

Material e Métodos: Estudo observacional prospetivo, envolvendo os doentes internados na unidade de cuidados intensivos pediátricos com depressão do estado de consciência, durante um ano. Ambas as escalas foram aplicadas diariamente aos doentes, por três avaliadores (médicos especialistas, médicos internos e enfermeiros), desde a admissão até à alta clínica, e as sequelas neurológicas foram avaliadas através da escala King's Outcome Scale for Childhood Head Injury - KOSCHI.

Resultados: Foram incluídos 27 doentes com idades compreendidas entre um e 17 anos. Ambas as escalas são fiáveis e a concordância inter-avaliador foi superior na escala FOUR. A escala de coma de Glasgow apresentou pontuação mínima em oito avaliações. No entanto, a escala FOUR apresentou o score mínimo em apenas duas dessas avaliações. Em ambas as escalas verificou-se uma forte associação entre o valor obtido à entrada e a evolução clínica do doente (escala FOUR: área sob a curva = 0,939; escala de coma de Glasgow: área sob a curva =0,925).

Discussão: A escala FOUR fornece mais informação sobre o estado neurológico dos doentes do que a escala de coma de Glasgow e tem interesse prognóstico.

Conclusão: A escala FOUR pode ser aplicada nos doentes internados com depressão do estado de consciência na unidade de cuidados intensivos pediátricos. Consideramos que um estudo multicêntrico seria importante para confirmar estes resultados.

Palavras-chave: Alterações da Consciência; Coma/diagnóstico; Criança; Escala de Coma de Glasgow; Tradução; Unidades de Cuidados Intensivos Pediátricos
\end{abstract}

\section{ABSTRACT}

Introduction: The Full Outline of UnResponsiveness - FOUR scale has been previously validated to assess impaired consciousness in the adult population. The aim of this study is the translation into Portuguese and validation of the FOUR scale in the pediatric population. The study also compares the FOUR scale and Glasgow coma scale score ratings and the clinical outcome of patients hospitalized in Pediatric Intensive Care Units.

Material and Methods: This study prospectively rated patients admitted to the Pediatric Intensive Care Units with impaired consciousness during one year. Both scales were applied daily to patients by three types of examiners: intensivists, residents and nurses, from the moment of admission until clinical discharge. Neurological sequelae was evaluated using the King's Outcome Scale for Childhood Head Injury - KOSCHI.

Results: Twenty seven patients between one and 17 years of age were included. Both scales are reliable and inter-rater reliability was greater for the FOUR score. Glasgow coma scale showed a minimum score in eight evaluations, whereas the FOUR scale obtained the minimum score in only two of these evaluations. In both scales there was a strong association between the admission score and the patient's outcome (area under curve FOUR $=0.939$, versus Glasgow coma scale $=0.925$ ).

Discussion: The FOUR scale provides more neurological information than Glasgow coma scale in patients with impaired consciousness and has prognostic interest.

Conclusion: The FOUR scale can be applied in patients admitted with impaired consciousness in Pediatric Intensive Care Units. We think that a multicenter study would be very beneficial for confirming and generalizing these results.

Keywords: Child; Coma/diagnosis; Consciousness Disorders; Glasgow Coma Scale; Intensive Care Units, Pediatrics Translating

\section{INTRODUÇÃO}

A escala de coma de Glasgow (GCS) foi publicada por Teasdale e Jennett em 1974, na revista Lancet, como um apoio para a avaliação da evolução clínica dos doentes com diminuição do estado de consciência e grau de profundidade do coma. ${ }^{1}$ Inicialmente, foi desenvolvida como um instrumento de pesquisa em doentes com traumatismo craniano grave, com o objetivo de melhorar a comu- nicação entre profissionais. ${ }^{1}$ Paulatinamente, foi aprovada a nível mundial como instrumento importante na avaliação de vítimas de trauma, tornando-se parte integrante dos cursos de suporte avançado de vida do American College of Surgeons. ${ }^{2}$ A GCS tem sido utilizada para diversos fins: classificação de estados de consciência, comparação da eficácia de tratamentos e indicação prognóstica.

1. Serviço de Pediatria Médica. Centro Hospitalar Vila Nova de Gaia/Espinho. Gaia. Portugal.

2. Serviço de Pediatria Médica. Centro Hospitalar do Porto. Porto. Portugal.

3. Laboratório de Biomatemática. Faculdade de Medicina. Universidade de Lisboa. Lisboa. Portugal.

4. Unidade de Cuidados Intensivos Pediátricos. Departamento de Pediatria. Hospital de Santa Maria. Centro Académico de Medicina de Lisboa. Lisboa. Portugal.

$\triangle$ Autor correspondente: Sofia Simões Ferreira. sofiaferreira20@gmail.com

Recebido: 20 de julho de 2016 - Aceite: 19 de abril de 2017 | Copyright @ Ordem dos Médicos 2017 
Um exemplo da sua utilização como indicador para o tratamento de patologia neurológica é a recomendação de intubação endotraqueal para proteção da via aérea, em doentes com valor de GCS igual ou inferior a oito. ${ }^{3} \mathrm{~A}$ popularidade desta escala deve-se à sua rápida e fácil aplicação, elevada reprodutibilidade inter-observador e demonstração do seu valor prognóstico em múltiplas causas de alteração do estado de consciência.

No entanto, a GCS apresenta várias limitações e desvantagens.

Como a resposta verbal não pode ser avaliada em doentes intubados existe a possibilidade de inconsistências no valor atribuído nesta categoria, isto é, os profissionais de saúde tanto atribuem o valor mínimo possível, como podem extrapolar o valor desta categoria, em função da presença de outros sinais neurológicos.

A GCS não inclui indicadores importantes para uma avaliação adequada da gravidade do coma, isto é, avalia apenas a função cortical, não incluindo parâmetros da avaliação da função do tronco cerebral, tais como o padrão respiratório, o tamanho e os reflexos pupilares e os movimentos oculares. Porém, a GCS avalia apenas os dois últimos, podendo não detetar devidamente a profundidade do coma. ${ }^{4}$ Esta escala tende a ser melhor aplicada por profissionais experientes e a interpretação de valores intermédios é complicada mesmo para os médicos intensivistas. ${ }^{5}$ Tendo em conta as limitações da GCS, surge a necessidade de desenvolver novas escalas de avaliação do nível de consciência, que providenciem maior detalhe do estado neurológico, sejam igualmente fáceis de aplicar e tenham valor prognóstico.

Uma das escalas que tem demonstrado melhores resultados é a escala FOUR, acrónimo de Full Outline of UnResponsiveness. Inicialmente desenvolvida na Mayo Clinic College of Medicine,$^{5}$ esta escala popularizou-se e é atualmente utilizada para a população adulta em vários centros de neurotrauma e unidades de cuidados intensivos (UCI) por todo o Mundo.

A escala FOUR (Tabela 1) consiste num teste que engloba quatro componentes: resposta ocular, resposta motora, reflexos de tronco cerebral e respiração. Cada componente é pontuado de zero a quatro $(0=$ pior, $4=$ melhor $) .{ }^{5}$

O acrónimo desta escala, FOUR, representa os quatro itens em análise e a pontuação máxima de quatro pontos, em cada um desses itens.

A resposta ocular permite distinguir um estado vegetativo de uma síndrome de locked-in, pois no primeiro, o doente pode ter os olhos abertos mas não seguir um objeto e, no segundo, ter os olhos abertos e piscar ou seguir um objeto a pedido. ${ }^{6} \mathrm{O}$ mesmo não se verifica na GCS que apenas avalia a abertura dos olhos.

A resposta motora inclui três movimentos diferentes das mãos (levantar o polegar, punho fechado ou sinal da paz), já validados e fiáveis para avaliar o nível de alerta. Inclui um

Tabela 1 - Escala FOUR. Composta por quatro componentes e respetivas pontuações

\begin{tabular}{l} 
(A) Resposta ocular \\
\hline 4 = Pálpebras abertas espontaneamente ou pelo examinador, segue o objeto ou pisca os olhos a pedido \\
3 = Pálpebras abertas, mas não segue objeto com o olhar \\
2 = Pálpebras fechadas, mas abre com estímulo de voz alta \\
1 = Pálpebras fechadas, mas abre com estímulo doloroso \\
0 = Pálpebras permanecem fechadas mesmo após estímulo doloroso
\end{tabular}

\section{(B) Resposta motora}

4 = Levanta os polegares ou os punhos ou faz sinal de paz

$3=$ Localiza a dor

2 = Flexão em resposta a estímulo doloroso

1 = Extensão em resposta a estímulo doloroso

$0=$ Sem resposta a estímulo doloroso ou mioclonias generalizadas

(C) Tronco cerebral

4 = Reflexos pupilares e corneanos presentes

3 = Uma pupila dilatada e fixa

2 = Reflexos pupilares ou corneanos ausentes

1 = Reflexos pupilares e corneanos ausentes

$0=$ Reflexos pupilares, corneanos e da tosse ausentes

\section{(D) Respiração}

4 = Não intubado, padrão respiratório regular

3 = Não intubado, padrão respiratório de Cheyne-Stokes

2 = Não intubado, respiração irregular

1 = Respira acima da frequência do ventilador

0 = Apneia ou respira na frequência do ventilador 
sinal de mau prognóstico após ressuscitação de paragem cardiorespiratória, as mioclonias generalizadas. Devido à dificuldade em distinguir a resposta de retirada à dor da flexão a um estímulo doloroso na GCS, as duas combinam-se na nova escala. ${ }^{5}$

Os dois componentes seguintes surgiram para substituir a resposta verbal da GCS que não é aplicável nos doentes intubados e que na população pediátrica tem de ser modificada para os doentes que ainda não adquiriram a linguagem.

O terceiro componente testa três reflexos do tronco cerebral: o reflexo pupilar, corneano e da tosse. Deste modo, permite avaliar o mesencéfalo, a protuberância e o bulbo raquidiano. Ao incluir a observação de pupila dilatada unilateral, a escala FOUR deteta lesão aguda do terceiro par craniano, sinal de compressão do tronco cerebral facilmente detetado através da visualização das pupilas e que implica intervenção imediata. O reflexo da tosse está normalmente ausente quando os reflexos pupilar e corneanos estão igualmente ausentes. ${ }^{5}$

Por fim, o padrão respiratório permite avaliar se o doente necessita de ventilação. A respiração Cheyne-Stokes e a respiração irregular indicam lesão bihemisférica ou lesão do tronco cerebral. Se o doente já estiver ventilado e a respirar acima da frequência do ventilador, significa que os centros de controlo da respiração estão funcionantes. ${ }^{5}$

Caso todas as categorias tenham a pontuação mínima, deve-se considerar a realização de provas de morte cerebral. $^{5}$

A escala FOUR é, de acordo com os autores da escala, simples de aplicar. É específica na deteção de determinados estados de depressão da consciência, como a distinção da síndrome de locked-in e do estado vegetativo, e fornece detalhes importantes acerca da função do tronco cerebral, que constitui uma avaliação necessária em doentes com alterações do estado de consciência. ${ }^{5}$

O objetivo deste estudo prospetivo consiste na tradução, validação e aplicação clínica da escala FOUR aos doentes internados numa unidade de cuidados intensivos pediátricos (UCIP), bem como na comparação dos resul- tados obtidos entre a escala FOUR e a GCS e sua relação com a previsão de mortalidade e morbilidade à alta hospitalar.

\section{MATERIAL E MÉTODOS}

Estudo observacional prospetivo, onde foram incluídas as crianças internadas numa UCIP, desde maio de 2012 a maio de 2013, com diminuição do estado de consciência (GCS inferior a 15) à admissão, e excluídas as crianças com diminuição de consciência, por doença crónica e as crianças sob o efeito de relaxantes neuromusculares.

Participaram no estudo enfermeiros, médicos internos e médicos especialistas de uma UCIP. Antes do início do estudo, foi realizada uma sessão de apresentação da escala FOUR para os avaliadores, aos quais se forneceu um documento com a escala FOUR, as suas instruções e também uma ficha para registo da avaliação de ambas as escalas. Posteriormente, realizou-se uma sessão de esclarecimento de dúvidas e uma segunda formação na aplicação da escala FOUR.

Para que o estudo se aproximasse o mais possível da prática clínica diária, cada doente foi avaliado diariamente pelo enfermeiro, médico especialista e médico interno responsáveis pelo doente, independentemente da experiência profissional do avaliador. Dependendo do estado clínico, determinados doentes foram submetidos a mais do que uma observação diária, por cada avaliador. As avaliações foram feitas com um intervalo máximo de uma hora entre os avaliadores, para assegurar que estes não tivessem conhecimento dos resultados dos outros avaliadores, e reduzindo a possibilidade de o estado clínico do doente se alterar. Aos doentes intubados foi atribuída a pontuação mínima na resposta verbal da GCS. Aos doentes com idade inferior a dois anos, que apresentassem movimentos espontâneos harmoniosos, no componente motor de ambas as escalas, ficou ao critério dos avaliadores atribuir a pontuação máxima ou a pontuação imediatamente abaixo.

Ao longo do período de recolha de dados, documentou-se o estado neurológico dos doentes à alta hospitalar, para posterior análise da relação entre as avaliações obtidas

Tabela 2 - Escala KOCHI (King's Outcome Scale for Childhood Head Injury)

\begin{tabular}{|c|c|}
\hline Pontuação 1 & Morte \\
\hline Pontuação 2 & Estado vegetativo \\
\hline Pontuação 3: Incapacidade grave & $\begin{array}{l}\text { a) A criança é capaz de, pelo menos, movimentar de um modo intermitente parte do corpo/ } \\
\text { olhos espontaneamente ou a pedido } \\
\text { b) Implica grande nível de dependência, mas a criança é capaz de realizar algumas tarefas } \\
\text { diárias }\end{array}$ \\
\hline Pontuação 4: Incapacidade moderada & $\begin{array}{l}\text { a) A criança é praticamente independente mas tem necessidade de algum grau de } \\
\text { supervisão parental para a realização de atividades diárias } \\
\text { b) A criança é independente de acordo com a idade, mas apresenta alguns distúrbios } \\
\text { residuais no que concerne à aprendizagem/ comportamento ou sequelas neurológicas }\end{array}$ \\
\hline Pontuação 5: Boa recuperação & $\begin{array}{l}\text { a) Nova condição patológica que não interfere com o funcionamento global da criança (ex. } \\
\text { cefaleias) } \\
\text { b) Recuperação total, sem sequelas detetáveis }\end{array}$ \\
\hline
\end{tabular}


na escala FOUR e GCS, e o prognóstico dos doentes em estudo, através da classificação de King's Outcome Scale for Childhood Head Injury (KOSCHI), Tabela 2.

A escala FOUR foi traduzida por uma empresa de serviços de tradução e posteriormente revista por dois médicos especialistas em Pediatria.

\section{Análise estatística}

Para avaliar a concordância entre os pares de avaliadores, em ambas as escalas, calcularam-se os valores de kappa de Cohen ( $\mathrm{k}$ : 1) em cada par de avaliadores, isto é, médico especialista/médico interno (ME/MI), médico especialista/enfermeiro (ME/E), médico interno/enfermeiro (MI/E); 2) em cada componente das escalas; 3 ) nos totais de cada avaliação. Este coeficiente permite avaliar a concordância das avaliações entre cada par de avaliadores, sendo que, para valores acima de 0,8 , se considera uma concordância excelente.

Para o cálculo de k para a pontuação total, na escala FOUR, procedeu-se à categorização desta variável em: Grave (pontuação total $\leq 7$ ), Moderado (pontuação entre 8 e 14) e Ligeiro (pontuação entre 15 e 16).

$\mathrm{Na}$ escala GCS ( $\mathrm{n}=82$ ) também foi efetuada a categorização da variável pontuação total em: Grave (pontuação total $\leq 8$ ), Moderado (pontuação entre 9 e 12) e Ligeiro (pontuação entre 13 e 14).

Relativamente à avaliação da consistência interna das escalas FOUR e GCS, utilizou-se o alfa de Cronbach, considerando-se uma escala fiável quando os valores de alfa são superiores a 0,8 .

Por fim, através do coeficiente de correlação de Spearman, determinou-se a correlação entre as duas escalas, averiguando-se se a escala FOUR permite uma avaliação dos doentes, no contexto pediátrico, como acontece com a escala GCS. Para a validação FOUR considerou-se uma amostra na qual estão incluídas todas as observações feitas pelos pares de avaliadores, para ambas as escalas $(n=$ 82).

Para comparar os resultados obtidos entre as duas escalas e a sua relação com a previsão de mortalidade e morbilidade à alta hospitalar, considerou-se a amostra constituída pela primeira observação de cada indivíduo $(n=27)$. Efetuou-se o cálculo da correlação entre cada uma das escalas com a KOSCHI, através do coeficiente de correlação de Spearman. Posteriormente, procedeu-se à análise da sensibilidade e especificidade, assim como ao cálculo da associação entre a pontuação obtida pelo doente na admissão na UCIP e a sua evolução clínica. A pontuação do doente à admissão na UCIP foi classificada em Gravidade / Não Gravidade. Esta classificação foi realizada para ambas as escalas: FOUR 0 - 8 (Gravidade) e 9 - 16 (Não Gravidade); 8 GCS 3 - 9 (Gravidade) e 10 - 15 (Não Gravidade).

Para as pontuações obtidas na admissão na UCIP e a evolução clínica de cada doente participante, calculou-se a curva ROC e a respetiva área sob a curva (AUC), para ambas as escalas e para cada classe de observador. Esta mesma análise foi também utilizada para correlacionar a pontuação obtida pelo doente à entrada na UCIP e a previsão da sua mortalidade.

Os dados obtidos neste estudo foram analisados com recurso ao programa informático IBM SPSS ${ }^{\circledR}$ versão 21.

\section{Inquérito aos avaliadores}

Após a conclusão do estudo, solicitou-se aos avaliadores que avaliassem a escala. Forneceu-se um inquérito, com cinco questões:

1) A escala FOUR é clinicamente relevante e fácil de aplicar;

2) A escala FOUR pode ser aplicada em poucos minutos;

3) A escala FOUR é mais completa na avaliação do doente em comparação com a escala de Glasgow;

4) A escala FOUR é superior à escala de Glasgow na avaliação da profundidade do coma e deteção de agravamento do estado do doente;

5) A escala FOUR é uma escala de avaliação de coma que eu aplicaria caso fosse geralmente aceite.

Estas questões foram analisadas estatisticamente com o objetivo de averiguar a aplicabilidade da escala FOUR, no contexto da prática clínica diária, segundo os profissionais que a utilizam.

\section{RESULTADOS}

Neste estudo observaram-se os 27 doentes que, durante o período de recolha de dados, foram internados na UCIP por alteração do estado de consciência e que preenchiam os critérios de inclusão do presente estudo observacional.

Considerou-se uma observação quando ambas as escalas foram aplicadas pelo médico especialista, médico interno e enfermeiro. Uma vez que cada doente foi observado diariamente ao longo do internamento na UCIP e, por vezes, mais do que uma vez, obtiveram-se no total 82 observações. Em cada observação, ambas as escalas foram aplicadas três vezes. No entanto, em algumas observações, as escalas foram aplicadas por apenas dois avaliadores, o que resultou num total de 221 aplicações para cada escala.

A idade média dos doentes foi de 9,5 anos (mediana 11 anos), com idades compreendidas entre um e 17 anos. Para o sexo masculino, a idade média foi de 7,7 anos e para o sexo feminino de 13,2 anos. Cinco doentes tinham menos de dois anos.

Quanto à etiologia/causa da alteração do estado de consciência, 16 doentes sofreram traumatismo crânio-encefálico, dois apresentavam encefalopatia hipóxico-isquémica por afogamento e os restantes foram distribuídos por síndrome hemolítico-urémico, acidente vascular cerebral hemorrágico, politraumatismo por atropelamento, secção medular $\mathrm{C} 0-\mathrm{C} 1$ por projétil de arma de fogo, malformação arterio-venosa cerebral, hidrocefalia, meningite, encefalite herpética e eclâmpsia. Dos 27 doentes que participaram no estudo, 14 foram intubados.

Para a escala FOUR, a pontuação total máxima de 16 
foi observada em 34 de 221 avaliações (15,5\%). A pontuação total máxima foi atribuída mais frequentemente pelos enfermeiros (13 em 77 avaliações, 16,9\%).

Para a GCS, foi atribuída a pontuação total máxima em 15 de 221 avaliações (6,8\%). O componente verbal, difícil de avaliar nos doentes intubados, foi o que apresentou pontuação mínima com maior frequência, em 132 de 221 avaliações $(59,7 \%)$. À semelhança da escala FOUR, a pontuação total máxima da GCS foi atribuída mais vezes
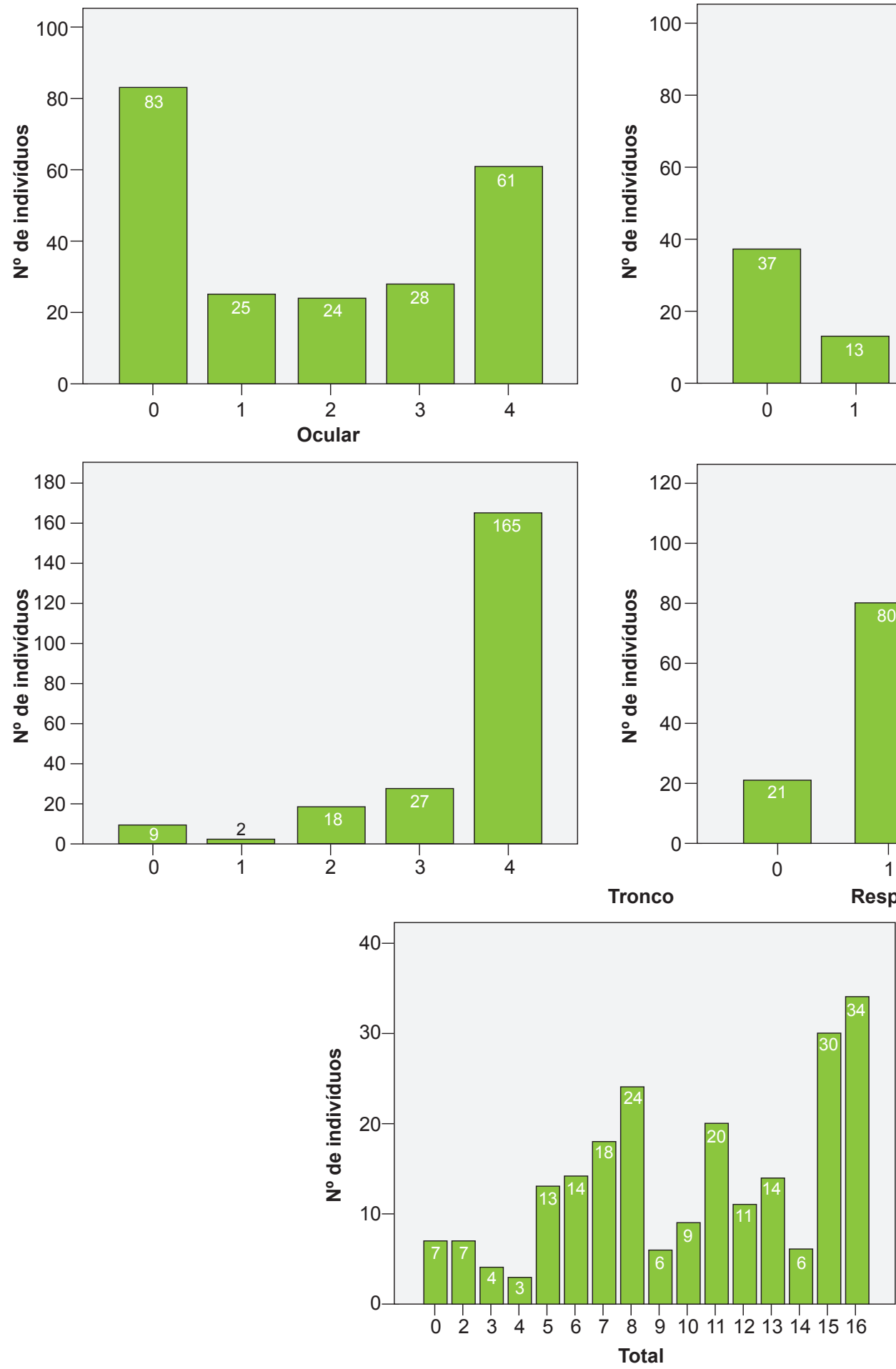

Figura 1 - Frequência de cada pontuação da escala FOUR

elos enfermeiros (sete em 78 avaliações, 9\%).

A frequência dos scores para cada escala encontra-se nas Fig.s 1 e 2.

O score mínimo da GCS, três, foi detetado em oito avaliações. Dessas oito avaliações, a escala FOUR apresentou o score mínimo em apenas duas avaliações.

O coeficiente de correlação Spearman entre a escala FOUR e a GCS foi muito elevado $(S p=0,954$ para $n=82)$.
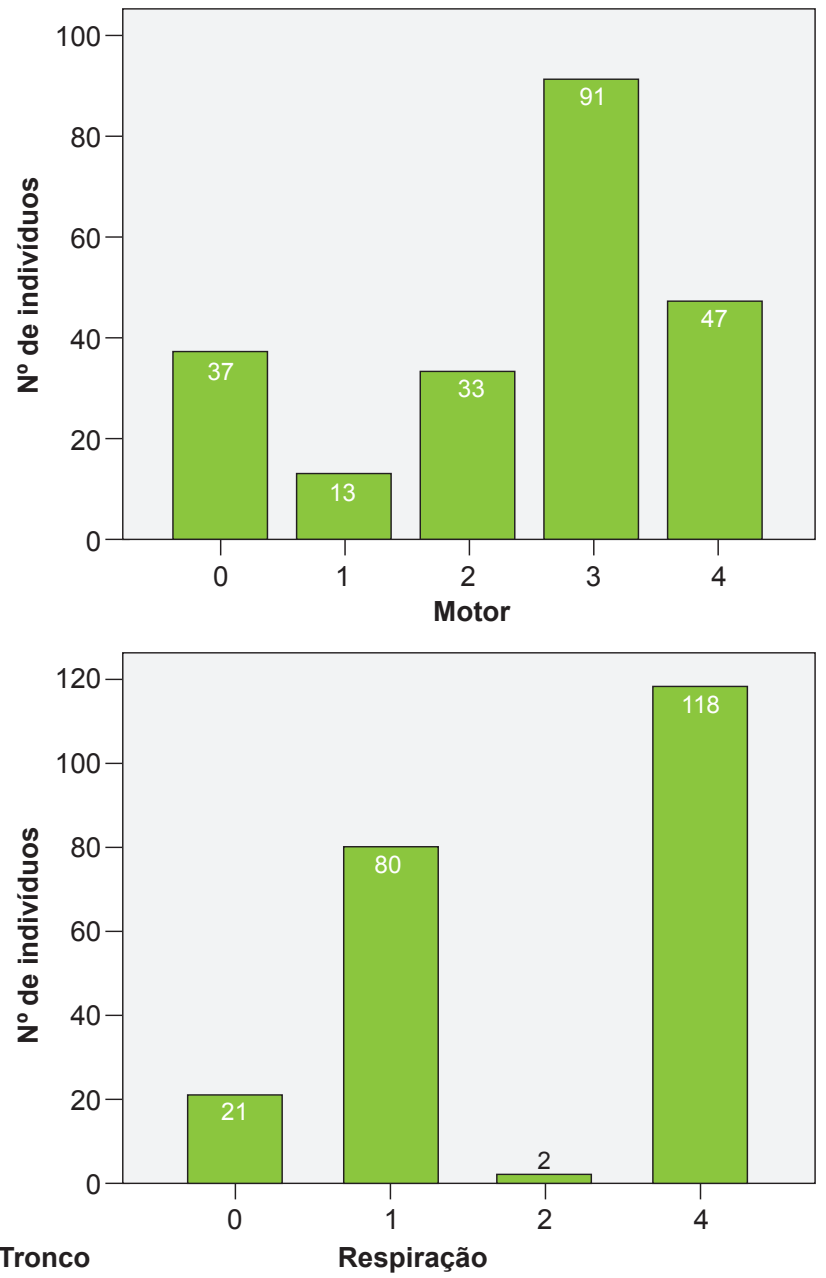

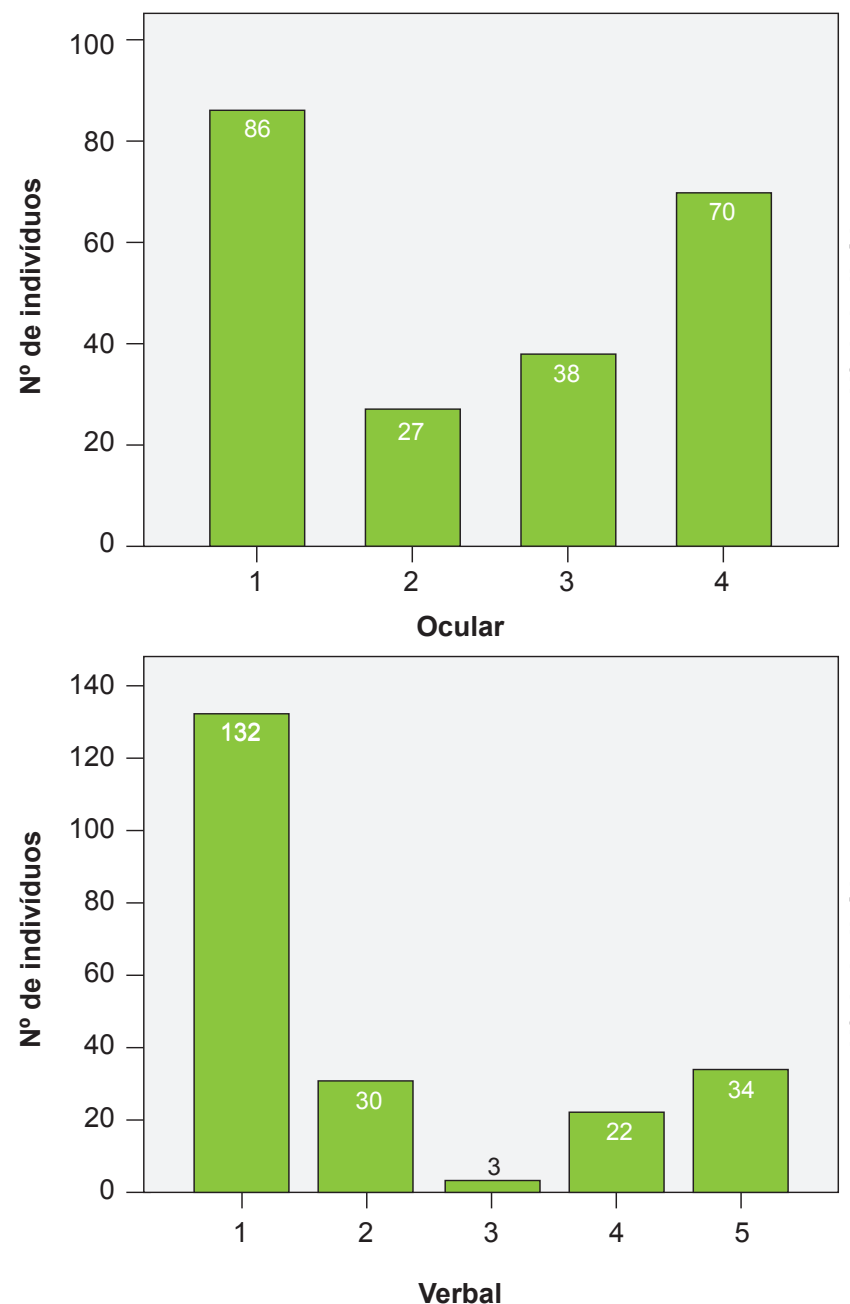

Figura 2 - Frequência de cada pontuação da escala GCS

Consistência interna / Replicabilidade interobservador

Quanto à análise da consistência interna, através do alfa de Cronbach, obteve-se um valor de 0,834 para a escala FOUR e 0,784 para a GCS.

$\mathrm{Na}$ escala FOUR, o valor do coeficiente de kappa de Cohen, no total de cada avaliação, para os pares de avaliadores $\mathrm{ME} / \mathrm{MI}, \mathrm{ME} / \mathrm{E}$ e $\mathrm{MI} / \mathrm{E}$ foi: 0,649, 0,757 e 0,806, respetivamente

Na GCS, o $\mathrm{k}$ do total de cada avaliação, para os pares de avaliadores ME/MI, ME/E e MI/E foi: 0,521, 0,536 e 0,767 , respetivamente.

A concordância entre os pares de avaliadores, em rela-
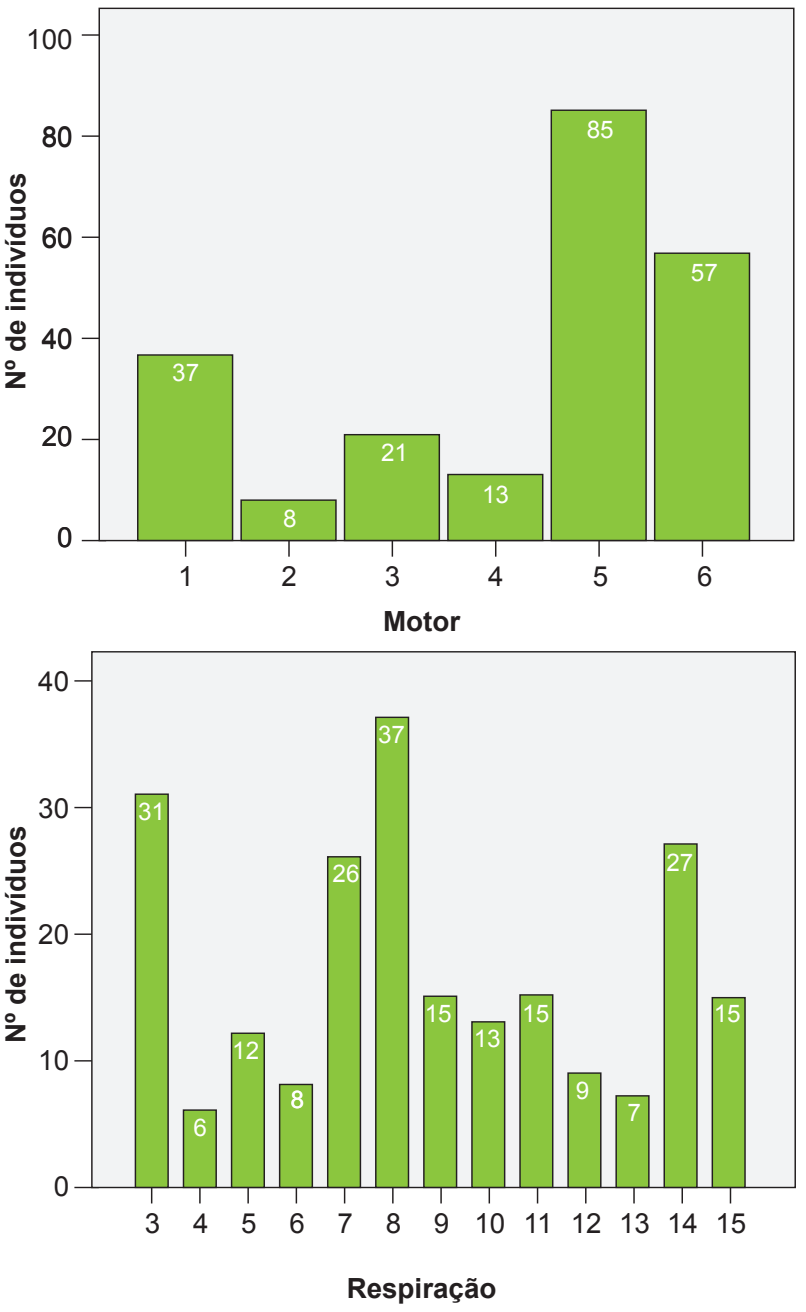

ção a cada componente de ambas as escalas, encontra-se na Tabela 3.

\section{Valor prognóstico}

$\mathrm{Na}$ amostra em estudo, cinco doentes morreram $(18,5 \% ; \mathrm{KOSCHI}=1) . \grave{A}$ alta hospitalar, apenas foi atribuído um doente em cada uma das categorias $3 \mathrm{~A}, 3 \mathrm{~B}, 4 \mathrm{~A}$ e $4 \mathrm{~B}$ da escala $\mathrm{KOSCHI}$. Três doentes obtiveram $\mathrm{KOSCHI}=5 \mathrm{~A}$ (boa recuperação) e $15 \mathrm{KOSCHI}=5 \mathrm{~B}$ (recuperação total). O coeficiente de correlação de Spearman é semelhante entre as escalas e o $\mathrm{KOSCHI}(\mathrm{Sp} \mathrm{GCS}=0,776$ contra Sp FOUR $=0,765$ ), revelando-se ambas as correlações fortes.

Tabela 3 - Concordância inter-avaliador para a escala FOUR e GCS

\begin{tabular}{|c|c|c|c|c|c|c|c|c|c|c|}
\hline \multicolumn{11}{|c|}{ Kappa de Cohen } \\
\hline \multicolumn{4}{|c|}{ Escala FOUR } & \multicolumn{7}{|c|}{ GCS } \\
\hline & $n$ & Ocular & Motora & $\begin{array}{l}\text { Tronco } \\
\text { cerebral }\end{array}$ & Respiração & Total & Ocular & Motora & Verbal & Total \\
\hline ME/MI & 61 & 0,590 & 0,575 & 0,757 & 0,859 & 0,649 & 0,569 & 0,587 & 0,733 & 0,521 \\
\hline ME/E & 62 & 0,612 & 0,492 & 0,848 & 0,809 & 0,757 & 0,573 & 0,604 & 0,747 & 0,536 \\
\hline MI/E & 73 & 0,637 & 0,638 & 0,719 & 0,784 & 0,806 & 0,634 & 0,681 & 0,816 & 0,767 \\
\hline
\end{tabular}


$\mathrm{Na}$ Tabela 4 apresentam-se os valores da sensibilidade e especificidade das escalas, assim como a associação entre o estado clínico do doente e a pontuação atribuída em cada uma das escalas e para cada tipo de observador. Os valores de especificidade obtidos para a escala em estudo foram superiores comparativamente aos valores da GCS. Em relação aos valores de sensibilidade foram mais elevados para a GCS. Os valores dos coeficientes de contingência (CC) quantificaram as associações entre a pontuação da escala e o prognóstico do doente, revelando ser substancialmente mais relevantes na escala FOUR.

Estes resultados foram obtidos tendo por base a classificação em Gravidade / Não Gravidade (a partir da recodificação da variável média das pontuações dos três avaliadores) e Boa Evolução Clínica / Má Evolução Clínica (sendo que o primeiro critério se aplica a valores de KOS$\mathrm{CHI}$ iguais ou superiores a quatro e, o segundo, a valores de $\mathrm{KOSCHI}$ inferiores a quatro).

Para um melhor conhecimento do desempenho das escalas como sistemas de classificação, calculou-se a curva ROC para ambas as escalas em estudo, assim como para as diferentes categorias de observadores. A Tabela 4 mostra os valores de AUC para cada observador, em cada uma das escalas. Este valor permite a comparação entre os desempenhos das escalas, utilizando a sensibilidade e especificidade.

Dos inquéritos distribuídos pelos avaliadores, 17 foram preenchidos. Verificou-se que 16 inquiridos $(94,1 \%)$ concordaram e um inquirido (5,9\%) não concordou que: a escala FOUR é clinicamente relevante e fácil de aplicar; a escala FOUR pode ser aplicada em poucos minutos; a escala FOUR é uma escala de avaliação de coma que o inquirido aplicaria caso fosse geralmente aceite. Catorze dos inquiridos $(82,4 \%)$ consideraram que a escala FOUR é mais completa na avaliação do doente em comparação com a escala de Glasgow. Por último, 15 inquiridos $(88,2 \%)$ consideraram que a escala FOUR é superior à escala de Glasgow na avaliação da profundidade do coma e na deteção de agravamento do estado do doente.

\section{DISCUSSÃO}

A escala FOUR foi desenvolvida com o propósito de colmatar algumas limitações da GCS, descritas anteriormente. ${ }^{5}$

A consistência interna das escalas foi boa na escala FOUR e aceitável na GCS.

Os avaliadores são profissionais de saúde de uma UCIP, que participaram independentemente dos anos de experiência, o que se aproxima mais da realidade da prática clínica, ao contrário de estudos feitos anteriormente.

Quanto à concordância entre os pares de avaliadores, na escala FOUR, os pares médico especialista / médico interno e médico especialista/enfermeiro alcançaram uma boa concordância. Já no par médico interno / enfermeiro, a concordância foi excelente. Na GCS a concordância foi razoável a moderada nos pares médico especialista / médico interno e médico especialista / enfermeiro e boa no par

Tabela 4 - Valores da sensibilidade, especificidade, resíduos ajustados, coeficiente de contingência obtidos para cada escala em estudo e valores de área sob a curva ROC (AUC)

\begin{tabular}{ccccccccc}
\hline \multicolumn{2}{c}{ FOUR } & \multicolumn{7}{c}{ GCS } \\
\hline & Total & ME & MI & E & Total & ME & MI & E \\
\hline S & 71,4 & 71,4 & 66,7 & 71,4 & 100,0 & 85,7 & 100,0 & 100,0 \\
E & 95,0 & 100,0 & 88,9 & 100,0 & 60,0 & 55,6 & 50,0 & 55,0 \\
RA & 3,6 & 4,0 & 2,7 & 4,2 & 2,7 & 1,9 & 2,2 & 2,5 \\
CC & 0,574 & 0,626 & 0,486 & 0,627 & 0,468 & 0,350 & 0,408 & 0,440 \\
\hline
\end{tabular}

S: sensibilidade; E: especificidade; RA: resíduos ajustados; CC: coeficiente de contingência; ME: médico especialista; MI: médico interno; E: enfermeiro; GCS: escala de coma de Glasgow

\begin{tabular}{lc}
\hline & Curva ROC (AUC) \\
\hline FOUR & \\
\hline Total & 0,939 \\
ME & 0,968 \\
MI & 0,907 \\
E & 0,943 \\
\hline GCS & \\
\hline Total & 0,925 \\
ME & 0,917 \\
MI & 0,875 \\
E & 0,921 \\
\hline
\end{tabular}


médico interno / enfermeiro. Trata-se de resultados notáveis, uma vez que, neste estudo, os avaliadores possuem pouca experiência com a nova escala e foram realizadas apenas duas sessões de formação, ao contrário do que acontece com a GCS, que é aplicada diariamente desde o início da atividade profissional dos avaliadores.

Quando este estudo foi iniciado, ainda não se encontravam disponíveis estudos publicados na população pediátrica. Entretanto foram surgindo estudos em que foram incluídas crianças a partir dos dois a cinco anos, com depressão do estado de consciência de causa traumática ou não traumática, que concluíram que a escala FOUR tem valor prognóstico superior ou sobreponível à GCS. No entanto, ainda são necessários mais estudos nesta população para determinar o valor prognóstico da escala FOUR e a sua aplicabilidade na pediatria. ${ }^{9-11}$

Os doentes nas UCl são muitas vezes medicados com fármacos sedativos, o que em doses médias, pode afetar a resposta motora e a abertura ocular, mas é menos provável que afete os reflexos do tronco cerebral ou a respiração. $\mathrm{Na}$ GCS, os três componentes são afetados pela sedação. . $^{6,12}$ No nosso estudo, a escala FOUR caracterizou melhor o estado de coma dos doentes que tiveram a pontuação mínima, três, na GCS. Tal como descrito em estudos anteriores, os componentes tronco cerebral e padrão respiratório forneceram informação adicional, sobre o estado neurológico nesses casos.

A GCS, ao incluir a resposta verbal, não foi tão vantajosa em cerca de metade dos doentes, por estarem intubados.

Os componentes tronco cerebral e padrão respiratório apresentaram concordâncias próximas ou mesmo excelentes nos três pares de avaliadores. O componente verbal da GCS manifestou uma boa concordância em todos os pares de avaliadores, provavelmente devido aos doentes intubados, aos quais foi atribuída a pontuação mínima.

A correlação entre cada uma das escalas FOUR e GCS e o $\mathrm{KOSCHI}$ revelou-se positiva e forte, o que indica que ambas evoluem no mesmo sentido, permitindo prever com idêntica eficácia a evolução dos doentes. Assim, este estudo vem também confirmar vários resultados de trabalhos realizados na população adulta, que postulavam a escala FOUR como a escala de avaliação do estado de consciência com maior valor preditivo na mortalidade intra-hospitalar e no estado clínico à alta hospitalar. ${ }^{4-6}$ As curvas ROC mostraram bons resultados nos dois sistemas de avaliação, com uma ligeira superioridade para a escala FOUR. Logo, há interesse clínico na utilização desta escala para classificar os doentes e definir o seu prognóstico.

De um modo geral, a escala FOUR obteve melhor associação entre a pontuação obtida e a mortalidade. A escala FOUR apresenta especificidade mais elevada, isto é, a probabilidade de não sobrevivência intra-hospitalar é maior para pontuações FOUR mais baixas, quando comparada com a GCS. A escala FOUR permite detetar sinais de agravamento do estado neurológico ao avaliar os reflexos do tronco cerebral (reflexo pupilar direto e consensual, reflexo corneano e reflexo da tosse), bem como o padrão respirató- rio. Este último componente permite igualmente identificar os doentes que podem necessitar de ventilação assistida ou, quando os doentes já se encontram ventilados, identificar os que apresentam centros de controlo da respiração funcionantes.

Este estudo apresenta algumas limitações. O número de doentes envolvidos foi reduzido (27 doentes) apesar de o número de observações ser razoável (82 observações). As crianças com menos de dois anos apresentam uma limitação no componente motor da escala FOUR, pois não são capazes de cumprir ordens. Contudo, esta limitação observa-se igualmente na GCS.

\section{CONCLUSÃo}

A escala FOUR pode ser aplicada na UCIP. É fácil de instruir e de aplicar. Fornece informações sobre o estado neurológico que permitem uma melhor avaliação do estado de consciência e, por conseguinte, um melhor encaminhamento do doente a partir do momento em que é admitido na UCIP. A escala FOUR deverá ser aplicada em crianças acima dos dois anos ou, em alternativa, poder-se-á desenvolver uma adaptação da escala para esta faixa etária, tal como foi desenvolvida para a GCS.

Este estudo pode ser considerado um estudo piloto no qual se faz uma primeira abordagem à validação da escala FOUR, com resultados positivos. Assim, será importante um estudo multicêntrico para confirmar e generalizar estes resultados e introduzir de uma forma mais generalizada a escala FOUR como uma boa alternativa à GCS na população pediátrica.

\section{PROTEÇÃO DE PESSOAS E ANIMAIS}

Os autores declaram que os procedimentos seguidos estavam de acordo com os regulamentos estabelecidos pelos responsáveis da Comissão de Investigação Clínica e Ética e de acordo com a Declaração de Helsínquia da Associação Médica Mundial. O estudo foi aprovado pela Comissão de Ética para a Saúde do Centro Hospitalar de Lisboa Norte/Faculdade de Medicina de Lisboa e autorizado pela Direcção Clínica do Centro Hospitalar de Lisboa Norte.

\section{OBSERVAÇÕES}

Este trabalho foi apresentado no $14^{\circ}$ Congresso Nacional de Pediatria, a 4 de outubro de 2013, no Porto.

\section{CONFIDENCIALIDADE DOS DADOS}

Os autores declaram ter seguido os protocolos do seu centro de trabalho acerca da publicação de dados.

\section{CONFLITOS DE INTERESSE}

Os autores declaram não terem qualquer conflito de interesse relativamente ao presente artigo.

\section{FONTES DE FINANCIAMENTO}

Os autores declaram não ter recebido subsídios ou bolsas para a elaboração do artigo. 


\section{REFERÊNCIAS}

1. Teasdale G, Jennett, B. Assessment of coma and impaired consciousness. A pratical scale. Lancet. 1974;2:81-4.

2. Sternbach GL. The Glasgow coma scale. J Emerg Med. 2000;19:67-71.

3. Chesnut RM. The management of severe traumatic brain injury. Emerg Med Clin North Am. 1997;15:581-604.

4. Kevric J, Jelinek GA, Knott J, Weiland TJ. Validation of the Full Outline of Unresponsiveness (FOUR) scale for conscious state in the emergency department: comparison against the Glasgow coma scale. J Emerg Med. 2011;28:486-90.

5. Wijdicks EF, Bamlet WR, Maramattom BV, Manno EM, McClelland RL. Validation of a new coma scale: The FOUR score. Ann Neurol. 2005;58:585-93.

6. Iyer VN, Mandrekar JN, Danielson RD, Zubkov AY, Elmer JL, Wijdicks EF. Validity of the FOUR score coma scale in the medical intensive care unit. Mayo Clin Proc. 2009;84:694-701.

7. Crouchman M, Rossiter L, Colaco T, Forsyth R. A practical outcome scale for paediatric head injury. Arch Dis Child. 2001;84:120-4.
8. Akavipat P. Endorsement of the FOUR score for consciousness assessment in neurosurgical patients. Neurol Med Chir. 2009;49:56571.

9. Kochar GS, Gulati S, Lodha R, Pandey R. Full Outline of UnResponsiveness score versus Glasgow coma scale in children with nontraumatic impairment of consciousness. J Child Neurol. 2014;29:1299-304.

10. Jamal A, Sankhyan N, Jayashree M, Singhi S, Singhi P. Full Outline of UnResponsiveness score and the Glasgow coma scale in prediction of pediatric coma. World J Emerg Med. 2017;8:55-60.

11. Cohen J. Interrater reliability and predictive validity of the FOUR score coma scale in a pediatric population. J Neurosci Nurs J Am Assoc Neurosci Nurses. 2009;41:261-9.

12. Wijdicks FM, Kramer AA, Rohs T Jr, Hanna S, Sadaka F, O'Brien J, et al. Comparison of the Full Outline of UnResponsiveness score and the Glasgow coma scale in predicting mortality in critically III patients. Crit Care Med. 2015;43:439-44. 